\section{Median raphe cyst: A clinically challenging diagnosis}

\author{
Chadi Hajar, Ibrahim R. Hajjali, \\ Laura Oscar, Daniel C. Mayes \\ Department of Pathology, Penrose-St. \\ Francis Health Services, Colorado \\ Springs, CO, USA
}

\begin{abstract}
Median raphe cyst is an uncommon developmental anomaly that can develop anywhere along the midline of the external genitals. Only a few hundred cases have been published in the English literature and the lack of awareness of this entity can lead to confusion and misdiagnosis. We report here a case of median raphe cyst located in the midline of the anterior scrotum of a 35 year-old man. Clinically, the patient presented with a scrotal mass increasing substantially in size over two days associated with tenderness, skin erythema, and scrotal pain. Radiologic interpretation of a sonogram and computed tomography scan suggested a thrombosed vessel. The patient was diagnosed with septic thrombophlebitis associated with overlying cellulitis. Despite conservative therapy with antibiotics, the patient developed pyrexia, tachycardia, and leukocytosis prompting surgical excision of the lesion. Histopathologic examination revealed an infected median raphe cyst. The cyst wall was lined by a stratified epithelium that included numerous Alcian blue positive goblet cells. The epithelial cells showed reactive changes with infiltration by numerous neutrophils. Our objective is to bring attention to and thereby facilitate the diagnosis of this unusual entity.
\end{abstract}

\section{Introduction}

Median raphe cyst is an uncommon anomaly that can develop at any location over the midline of the external genital region from the anus to the scrotum and perineum. This uncommon cystic lesion was first described by Mermet in $1985 .^{1}$ Only a couple hundred case reports of median raphe cysts have been published in the English literature describing cysts located over the glans penis, penile shaft, perineum, para meatus and scrotum. We report a new case of median raphe cyst located over the anterior scrotal area presenting as a painful scrotal mass.

\section{Case Report}

A 35-year-old male presented to the Emergency Department with scrotal pain. Approximately two years prior to this presentation, the patient reports first noticing a painless scrotal mass. Since that time, the mass had slowly grown. It has never caused him pain or other symptoms until this presentation. In the 2 days prior to seeking medical advice, the lesion became increasingly larger and more painful with a sensation of fullness, as described by the patient. The patient denied history of urologic trauma or infections. On exam, the scrotum had a $5 \mathrm{~cm}$ irregularly shaped, tubular-like structure at the midline, extending from the top of the scrotum to the base. There was surrounding erythema and warmth. This lesion was very tender to palpation, non-fluctuant, and without discharge. The bilateral testicles were normal without tenderness on palpation. There were no signs of Fournier's gangrene or deep space infection. The clinical impression was that of a mild scrotal cellulitis and possible thrombus of a scrotal varicosity $v s$ an inflamed scrotal cyst. The remainder of the physical exam was non-contributory. The patient underwent a sonogram (Figure 1) and computed tomography scan (Figure 2) that suggested the lesion was a thrombosed vessel. He was diagnosed in the emergency department with septic thrombophlebitis associated with overlying cellulitis and he was started on Vancomycin, Clindamycin and Rocephin. The patient failed conservative therapy with antibiotics and started to develop a fever of $102.6{ }^{\circ} \mathrm{F}$, tachycardia, and leukocytosis. Surgery was consulted and they elected to remove the lesion in the operating room.

Pathologic examination revealed a cyst wall lined by a stratified epithelium that included numerous Alcian blue and mucicarmine positive goblet cells (Figure 3C and D). The epithelial cells showed marked reactive changes with infiltration by numerous neutrophils (Figure 3A and B). The surrounding connective tissue showed hemorrhage, edema, and acute and chronic inflammation with a prominent neutrophilic infiltrate (Figure 3A and B). The overlying epidermis was unremarkable. Other sections showed an abscess just deep to the epidermis that was focally lined by flattened epithelium, but which had been denuded in many areas and attenuated in others. Squamous differentiation was present in some foci (Figure 3B). The Alcian blue/Periodic Acid-Shiff (PAS) and mucicarmine stains included concurrently performed controls showing appropriate reactions. The final pathologic diagnosis was an infected median raphe cyst.
Correspondence: Chadi Hajar, Department of Pathology, Penrose-St. Francis Heath Services, 2222 N. Nevada Ave., Colorado Springs, CO 80907, USA.

Tel. +1.719.776.5816 - Fax: +1.719.776.2108.

E-mail: chadihajar@centura.org

Key words: Median raphe cyst; Thrombophlebitis; Scrotum; Diagnosis.

Contributions: the authors contributed equally. Conflict of interest: the authors declare no potential conflict of interest.

Funding: none.

Received for publication: 30 May 2019

Revision received: 1 August 2019.

Accepted for publication: 30 August 2019

This work is licensed under a Creative Commons Attribution NonCommercial 4.0 License (CC BY-NC 4.0).

(C) Copyright: the Author(s), 2019

Licensee PAGEPress, Italy

Clinics and Practice 2019; 9:1176

doi:10.4081/cp.2019.1176

\section{Discussion and Conclusions}

Median raphe cysts are an uncommon benign congenital lesion. They are usually asymptomatic lesions detected in the first decade of life with symptomatic clinical presentations typically occurring in the second and third decades of life. ${ }^{2}$ In children, symptoms might include dysuria, frequency, redirected urinary stream, infection and hematuria. ${ }^{2}$ In adolescents and adults, hematospermia and difficulty in sexual function may be encountered. ${ }^{3}$ In some cases, the cyst may grow rapidly following a period of relative indolence and can even become inflamed or traumatized, complicating diagnosis. ${ }^{2,4,5}$ Raphe cysts are generally solitary and translucent, and can develop at any location over the midline of the external genitalia from the parameatal region to the perineum. ${ }^{1}$ The penile shaft is the more common location at presentation, regardless of patient age. ${ }^{6}$ This is likely due to the lesion being readily identified by direct inspection and the reported increase in symptoms associated with parameatal and penile lesions. ${ }^{2,6}$ The cysts are typically less than $2 \mathrm{~cm}$ in size, however scrotal cysts can be larger than lesions in other location., 2,6,7

Management of median raphe cysts will depend on the clinical presentation. Observation in asymptomatic patients is acceptable and spontaneous resolution of the lesion has been reported. ${ }^{3,6}$ For symptomatic patients or those seeking a cosmetic resolution, surgical excision with primary closure is the definitive treatment 


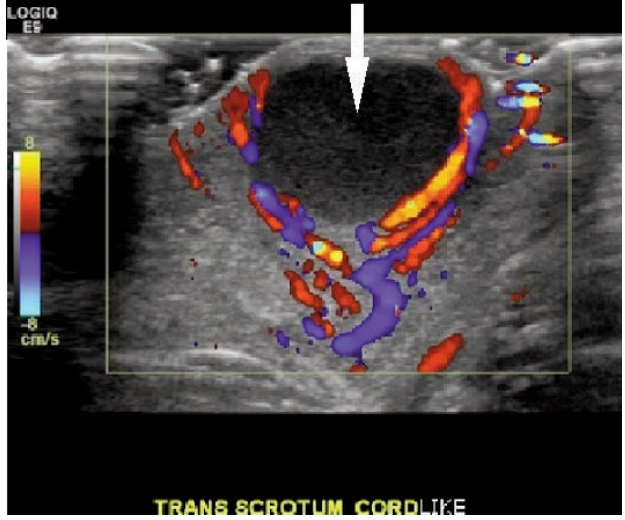

Figure 1. Ultrasound showing the $7.5 \times 1.5 \times 1.2 \mathrm{~cm}$ avascular cystic structure. Left hemiscrotum: testicular morphology and flow is normal. Along the posterior scrotum, extending from the base of the penis at least $8 \mathrm{~cm}$ is a $7.5 \times 1.5 \times 1.2 \mathrm{~cm}$ avascular fluid filled tubular structure with thickened walls (white arrow). Ultrasound impression was an unusual complex tubular structure in the superficial posterior scrotum, no associated mass, most likely occlusion of superficial vein.

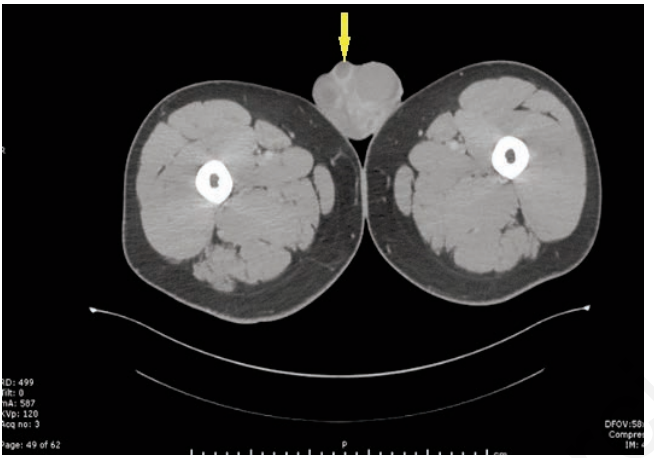

Figure 2. Computed tomography (CT) scan showing the scrotal cyst along the scrotal midline. Concordant with ultrasound, an avascular appearing tubular structure (yellow arrow) that extends from the base of the penis anterior and inferior along the scrotum. No overt findings of infection in the regional fat. CT impression was most likely a thrombosed vessel, consider urologic consultation.
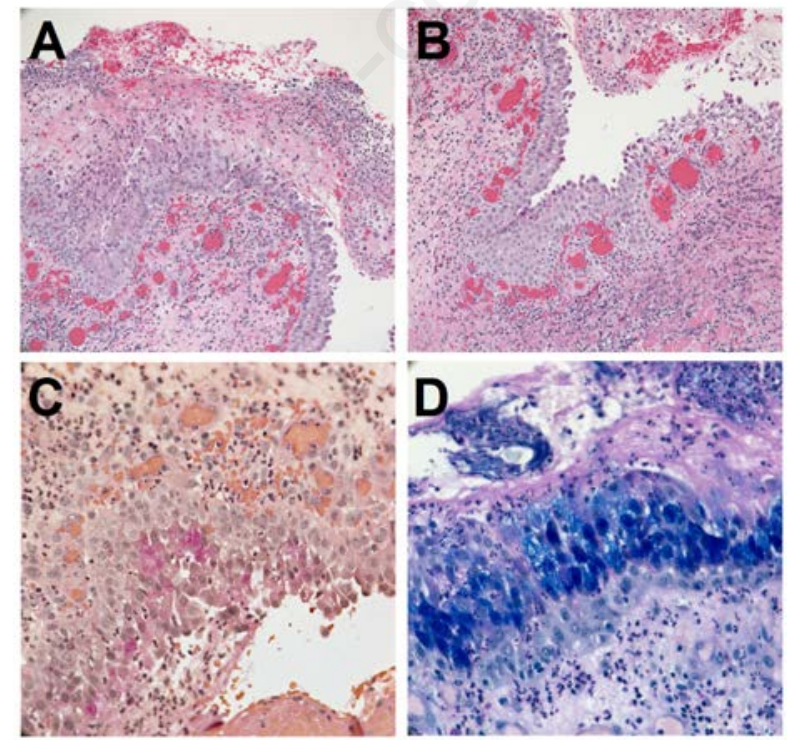

Figure 3. A) Transitional epithelium mixed with goblet cells and a prominent neutrophilic infiltration $(H \& E$ stain, $\times 200)$. B) Squamous epithelium lining with a prominent neutrophilic infiltration $(\mathrm{H} \& \mathrm{E}$ stain, $\times 200)$. C) Mucin producing goblet cells are highlighted in pink (Mucicarmine stain, $\times 400)$. D) Alcian blue/Periodic Acid-Shiff (PAS) also demonstrates the presence of goblet cells (Alcian Blue/PAS stain, $\times 400$ ). method. ${ }^{4,7}$ Marsupialization should be avoided whenever possible as this leads to an open sinus and cosmetically inferior outcomes. Aspiration of the cyst alone is insufficient as this is associated with recurrence. ${ }^{2,4,6-8}$ In our case, the patient presented with a swollen, painful and erythematous scrotal lesion diagnosed as thrombophlebitis by imaging studies. Although a median raphe cyst was not suspected, surgery was implicated to remove the lesion due to the lack of response to antibiotic therapy. Histologically, four different types have been described in median raphe cyst: urethral, epidermal, glandular and mixed. ${ }^{5}$ The cyst lining in our case was mixed, comprised of urotheliumlike epithelium mixed with scattered mucinous goblet cells and squamous cell epithelium. Alcian blue/PAS and muscarmine stains with appropriate controls were used to highlight the mucinous goblet cells.

The differential diagnosis for a median raphe cyst may include apocrine cystadenoma, epidermal inclusion cyst, pilonidal disease, and other genital cystic lesions. Imaging studies are not generally helpful in distinguishing between these and is generally reserved for more confounding clinical presentations. Histopathological findings and history are required to obtain the correct diagnosis.

\section{References}

1. Mermet P. Congenital cysts of the genitoperineal raphe. Rev Chir 1895;15:382435.

2. Shao IH, Chen TD, Shao HT, Chen HW Male median raphe cysts: serial retrospective analysis and histopathological classification. Diagn Pathol 2012;7:121.

3. Deliktas H, Sahin H, Celik OI, Erdogan O. Median raphe cyst of the penis. Urol $\mathrm{J}$ 2015;12:2287-8.

4. Syed MMA, Amatya B, Sitaula S. Median raphe cyst of the penis: a case report and review of the literature. J Med Case Rep 2019; $13: 214$

5. Park CO, Chun EY, Lee JH. Median raphe cyst on the scrotum and perineum. J Am Acad Dermatol 2006;55:S114-5.

6. Amaranthan A, Sinhasan SP, Dasiah SD. Median raphe cysts of the prepucial skin, with triple histological linings: a case report and review of the literature. J Clin Diagn Res 2013;7:1466-8.

7. Matsuyama S, Matsui F, Yazawa K, et al. Long-term follow-up of median raphe cysts and parameatal urethral cysts in male children. Urology 2017;101:99-103.

8. Navarro HP, Lopez PC, Ruiz JM, et al. Median raphe cyst. Report of two cases and literature review. Arch Esp Urol 2009;62:585-9. 